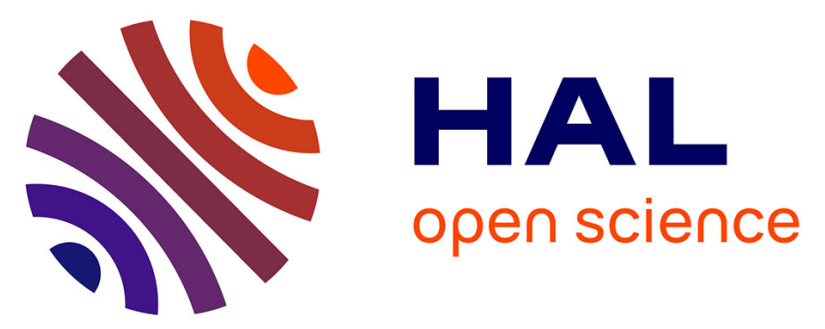

\title{
Evaluation of needle biopsy as a potential risk factor for local recurrence of Wilms tumour in the SIOP WT 2001 trial
}

Sabine Irtan, Harm van Tinteren, Norbert Graf, Marry M. van den Heuvel-Eibrink, Hugo Heij, Christophe Bergeron, Beatriz de Camargo, Tomas Acha, Filippo Spreafico, Gordan Vujanic, et al.

\section{To cite this version:}

Sabine Irtan, Harm van Tinteren, Norbert Graf, Marry M. van den Heuvel-Eibrink, Hugo Heij, et al. Evaluation of needle biopsy as a potential risk factor for local recurrence of Wilms tumour in the SIOP WT 2001 trial. European Journal of Cancer, 2019, 116, pp.13 - 20. 10.1016/j.ejca.2019.04.027 . hal-03486180

\section{HAL Id: hal-03486180 \\ https://hal.science/hal-03486180}

Submitted on 20 Dec 2021

HAL is a multi-disciplinary open access archive for the deposit and dissemination of scientific research documents, whether they are published or not. The documents may come from teaching and research institutions in France or abroad, or from public or private research centers.
L'archive ouverte pluridisciplinaire HAL, est destinée au dépôt et à la diffusion de documents scientifiques de niveau recherche, publiés ou non, émanant des établissements d'enseignement et de recherche français ou étrangers, des laboratoires publics ou privés.

\section{(ㅇ)(1) $\$$}

Distributed under a Creative Commons Attribution - NonCommerciall 4.0 International 


\section{Evaluation of needle biopsy as a potential risk factor for local recurrence of Wilms tumour in the SIOP WT 2001 trial.}

Sabine $\operatorname{Irtan}^{\mathrm{a}, \mathrm{b}}$, Harm Van Tinteren ${ }^{\mathrm{c}}$, Norbert Graf ${ }^{\mathrm{d}}$, Marry M. van den Heuvel-Eibrink ${ }^{\mathrm{e}}$, Hugo Heij $^{\mathrm{e}}$, Christophe Bergeron ${ }^{\mathrm{f}}$, Beatriz de Camargo ${ }^{\mathrm{g}}$, Tomas Acha ${ }^{\mathrm{h}}$, Filippo Spreafico ${ }^{\mathrm{i}}$, Gordan Vujanic $^{\mathrm{j}}$, Mark Powis ${ }^{\mathrm{k}}$, Bruce Okoye ${ }^{1}$, Jim Wilde $^{\mathrm{m}}$, Jan Godzinski ${ }^{\mathrm{n}}$, Kathy Pritchard-Jones ${ }^{\mathrm{a}}$

a. Cancer Section, Developmental Biology \& Cancer Programme, Institute of Child Health, University College London, London, UK. (k.pritchard-jones@ucl.ac.uk)

b. Paediatric Surgery Department, Trousseau Hospital - Assistance Publique des Hôpitaux de Paris, Paris, France

c. Biostatistics Department, Netherlands Cancer Institute - Antonie van Leeuwenhoekhuis Plesmanlaan, Amsterdam, Netherlands (h.v.tinteren@nki.nl)

d. Saarland University, Department of Pediatric Oncology \& Hematology, Homburg, Germany (Norbert.Graf@uniklinikum-saarland.de)

e. Princess Máxima Center for Pediatric Oncology, Utrecht, The Netherlands (m.m.vandenheuvel-eibrink@prinsesmaximacentrum.nl) (hugo.heij@icloud.com)

f. Pediatric Oncology Unit, Centre Léon Bérard, Lyon, France (christophe.bergeron@ihope.fr)

g. Pediatric Hematology and Oncology Program, Research Center, Instituto Nacional de Câncer, Rio de Janeiro, Brazil (bdecamar@terra.com.br)

h. Department of Pediatric Oncology, Hospital Materno-Infantil, Malaga, Spain (tachavalls@gmail.com)

i. Department of Clinical Oncology and Hematology, Pediatric Oncology Unit, Fondazione IRCCS Istituto Nazionale Tumori, Milan, Italy (filippo.spreafico@istitutotumouri.mi.it)

j. Department of Pathology, Sidra Medicine, Doha, Qatar (gvujanic@sidra.org)

k. Department of Paediatric Surgery, Leeds Teaching Hospitals NHS Trust, Leeds, UK (Mark.Powis@nhs.uk)

1. St. Georges Healthcare NHS Trust, Tooting, London, UK (bruce.okoye @ nhs.net)

m. Division of Pediatric Surgery, Geneva University Hospitals, University Center of Pediatric Surgery of Western Switzerland, Geneva, Switzerland (jim.wilde@hcuge.ch) 
n. Department of Paediatric Surgery, Marciniak Hospital, and Dept. of Paediatric Traumatology and Emergency Medicine, Medical University, Wroclaw, Poland (jgodzin@wp.pl)

\section{Correspondence to:}

Pr Sabine Irtan

Service de Chirurgie Pédiatrique Viscérale et Néonatale

Hôpital Trousseau - APHP

26 Avenue du Dr Arnold Netter

75012 Paris

France

Tel +33171738798

Fax + 33144736979

Sabine.irtan@gmail.com

Acknowledgements: The SIOP WT 2001 study was funded by Cancer Research UK (grant C1188/A8687), the UK National Cancer Research Network and Children's Cancer and Leukaemia Group (CCLG) (who supported the UK section), Société Française des Cancers de l'Enfant and Association Leon Berard Enfant Cancéreux and Enfant et Santé (who supported the French section), Gesellschaft für Pädiatrische Onkologie und Hämatologie and Deutsche Krebschilfe (grant 50-2709-Gr2, who supported the German section), Grupo Cooperativo Brasileiro para o Tratamento do Tumour de Wilms and Sociedade Brasileira de Oncologia Pediátrica (who supported the Brazilian section), the Spanish Society of Pediatric Haematology and Oncology and the Spanish Association Against Cancer (who supported the Spanish section) and the International Society of Pediatric Oncology coordinating centre in The Netherlands (SIOP-NL). K P-J is partly supported by the National Institute for Health Research Biomedical Research Centre Funding Scheme. We acknowledge the enormous efforts made by more than 1000 clinicians working at the 251 childhood cancer treatment centers in this study from 26 countries who enrolled and followed up patients in this study, and the patients and their families for their participation. 


\section{Abstract (247 words)}

Rationale: The impact of biopsying Wilms tumour (WT) at diagnosis on assigning tumour stage and recommended treatment remains controversial. To address this important question, we analyzed the potential association of all types of biopsy with local recurrence in patients treated in the SIOPWT2001 trial, where needle biopsy was permitted without 'upstaging' the tumour to stage III. Only open biopsy required treatment as stage III.

Methods: Among 2971 patients with unilateral WT (stages I-IV), 420 relapsed (139 local). Risk factors for recurrence were analyzed by Cox proportional hazard methods.

Results: Biopsy was performed in 969/2971 (33\%) patients (64\% cutting needle, 30\% fine needle aspiration (FNA), 6\% open biopsy). Biopsied patients were older, with larger tumours and a greater proportion with high-risk histology. In multivariate analysis (MVA) that included all factors associated with local recurrence in univariate analysis, only high-risk histology (Hazard ratio, HR=2.32; 95\%CI:1.58-3.42, $\mathrm{p}=<0.0001)$, age $\geq 2$ years $\quad(\mathrm{HR}=2.24$; 95\% CI:1.22-4.09, $\mathrm{p}=0.01)$ and preoperative tumour volume $(\mathrm{HR}=1.07 / 100 \mathrm{ml}$; 95\%CI 1.021.12, $\mathrm{p}=0.01$ ) were significant. The HR for the association of local recurrence, event-free and overall survival with biopsy was not significant $(\mathrm{HR}=1.4 ; 95 \% \mathrm{CI}$ : $0.9-2.17, \mathrm{p}=0.13 ; \mathrm{HR}=1.1$; 95\% CI:0.85-1.42, $\mathrm{p}=0.46$ and $\mathrm{HR}=1.13 ; 95 \% \mathrm{CI}: 0.79-1.62, \mathrm{p}=0.51$, respectively). These results were not materially different whether FNA or open biopsy were included in the biopsy group or not.

Conclusions: This post hoc analysis provides some reassurance that needle biopsy is not an independent adverse factor for either local recurrence or survival after adjustment for all relevant risk factors. Needle biopsy should not be an automatic criterion to 'upstage' WT.

Keywords: Wilms tumour, local relapse, biopsy, SIOP WT 2001 


\section{Introduction (2751 words)}

The clinical impact of biopsy on WT staging is controversial, due to debated results on the possible relationship between biopsy and the risk of local recurrence. Based on the results of the National Wilms Tumour Study (NWTS) 3-4 trials, showing that patients with stage II disease and local spillage had worse outcome than those with no spillage, the Children's Oncology Group (COG) AREN0532 protocol now considers any patient with local spillage to have a stage III tumour [1-2]. Any biopsy, regardless of type - fine needle aspiration (FNA), percutaneous cutting needle biopsy (PCNB or 'Tru-cut') or open surgery is considered as tumour spillage [2]. By contrast, the most recent trial of the International Society of Paediatric Oncology Renal Tumours Study Group (SIOP-RTSG, trial protocol SIOPWT2001) permitted tumour biopsy by FNA or PCNB without affecting tumour stage assignment, with the latter determined by the pathological stage of the nephrectomy specimen $[3]$.

In the UKW3 randomized trial, that compared immediate nephrectomy with a pre-operative chemotherapy approach, UK clinicians had routinely biopsied patients assigned to delayed nephrectomy using PCNB without affecting tumour staging [4]. Therefore, when the UK joined the SIOPWT2001 trial, this national group had continued their national practice of biopsying patients before starting chemotherapy [4-5]. However, concerns about a higher rate of local relapse in the UKW3 trial led to a post hoc analysis that showed equivocal results with an association between biopsy and local relapse on univariate analysis (UVA) that lost statistical significance on multivariable analyses after adjustment for other known risk factors for recurrence [6]. Interpretation was made more difficult by the small number of events and the fact that the biopsy group contained an excess of larger and more advanced stage tumours in older patients [6]. 
The SIOPWT2001 trial dataset provides a unique opportunity to analyze the potential association of biopsy as a risk factor for local recurrence or adverse survival outcome in a cohort of patients treated uniformly with a pre-operative chemotherapy approach, taking account in multivariable analysis (MVA) of the confounding factors that influence prognosis. We therefore conducted a retrospective post hoc analysis of the SIOPWT2001 trial database, using the same patient inclusion/exclusion criteria that had been applied to the UKW3 trial dataset, to test the hypothesis that biopsy may be an independent risk factor for local recurrence.

\section{Methods}

\section{Eligible patients}

Between 2001 and 2011, 3313 patients were registered in the SIOPWT2001 trial with localized or metastatic unilateral WT (Supplemental Figure 1). Thirty patients were excluded due to missing information on whether and how biopsy was performed. As the main outcome was risk factors for local recurrence, patients whose tumours were not treated by the standard approach of total unilateral nephrectomy after pre-operative chemotherapy $(n=312$, including 133 who had nephron-sparing surgery and one case of extra-renal tumour) were excluded. Metachronous relapse was not considered as recurrence as it is likely to represent a new primary tumour due to genetic predisposition. The final study population consisted of 2,971 patients.

The SIOPWT2001 trial was approved through relevant national ethical and regulatory processes in each country. All participants or their parents provided informed consent that included use of their clinical data for research. 


\section{Definition of biopsy}

Patients were categorized with respect to the "biopsy" variable recorded on the relevant case report form (CRF). The registration options were "no biopsy", FNA, PCNB or open (surgical) biopsy.

\section{Definition of tumour rupture}

This was taken from the CRFs where the presence of any rupture could be reported, regardless of its timing as pre- or peri-operatively.

\section{Classification of relapse}

Site of relapse was recorded on the CRFs as either 'primary site (tumour bed)', 'other abdominal site', 'lung', 'liver', or 'extra-abdominal site'. 'Primary site' and 'other abdominal site' were assumed to represent direct physical spread and were considered as "local relapse", as in the UKW3 analysis [6]. Abdominal relapse that was confined to the liver was categorized as 'distant' relapse due to haematogenous spread, and grouped together with relapse in the lung or other non-abdominal sites. "Combined" relapse was defined as a combination of local and distant relapse within the same patient.

\section{Analysis restricted to patients with low and intermediate risk stage I and II WT}

The use of doxorubicin and radiotherapy may negate the adverse effect of biopsy. To mitigate for this effect, we performed an analysis restricted to 797 patients with stage I and II, low and intermediate risk histology WT who received only vincristine and actinomycin D and no doxorubicin in both pre- and post-operative chemotherapy and no radiotherapy. This cohort excluded any patients documented to have had open biopsy, positive lymph nodes, tumour 
capsule rupture and those who received radiotherapy to the flank or 'high-risk' post-operative chemotherapy regimens.

\section{Statistical methodology}

Time to an event was calculated from the date of diagnosis. Survival curves were made using the Kaplan-Meier technique. Cox proportional hazard regression analysis was used to investigate the association of potential prognostic factors with each of the four outcome variables (local relapse, distant relapse, any event and overall survival). The very first event was considered and was either local relapse, distant relapse or a combination of both (any event). No death occurred without prior relapse. Overall survival was considered separately. Factors were tested for proportionality and linearity in case of continuous variables (age was categorised as 6 months to 1.99 years, 2 to 3.99 years and 4 and older). Results were expressed in terms of hazard ratio (HR), 95\% confidence interval (CI) and p-value. All multivariable models are stratified by 'national country'. For uni- and multivariable Cox proportional hazards models, missing values were imputed with either median value (continuous covariate) or with the most common value (categorical covariate) in the following covariates: rupture at surgery yes/no (22\% missing values), volume at diagnosis (13\% missing values) and volume at surgery (10\% missing values).

\section{Results}

\section{Clinical characteristics}

Among 2971 patients included in the analysis, 969 (33\%) had a biopsy, 288 (29.7\%) by FNA, $620(64.0 \%)$ by PCNB and $61(6.3 \%)$ by open surgery. $97 \%$ of UK patients had a biopsy 
compared to only $8 \%$ of German patients. All other participating countries had a global biopsy rate of around 20\%-26\% (Table 1). Only patients with open biopsy required treatment as stage III.

The 'no biopsy' and 'biopsy' groups exhibited some differences, with older patients, larger tumours at diagnosis and surgery and more patients with higher abdominal tumour stage and/or metastases in the 'biopsy' group (Table 2). There were slightly more high-risk histology tumours in the 'biopsy' group (15.8\% vs $12.3 \%)$ and features making a tumour stage III were also more frequent (Table 2). Pre-operative chemotherapy consisted of three drugs in $25 \%$ of patients being biopsied compared to $14 \%$ of patients in the 'no biopsy' group. A greater proportion of patients in the biopsy group had post-operative chemotherapy with the four-drug regimen (20\% versus 12\%) (Supplemental Table 1).

\section{Outcome}

At a median follow-up of 6 years, $420(14.1 \%)$ patients experienced a relapse, $86(20 \%)$ local, $222(53 \%)$ distant and $53(13 \%)$ combined. In 59 patients, the relapse site was not further specified (Table 3, Figures 1-2).

\section{Local relapse risk factors}

On univariable analysis of risk factors for local recurrence, the following were significantly associated with a higher risk: age $>2$ years, tumour volume at diagnosis and at surgery, histological risk group, abdominal tumour pathological stage III, positive lymph nodes (LN), tumour rupture, biopsy (yes/no) and type of biopsy (PCNB and open only) (Table 4).

On MVA, only age $>2$ years $(\mathrm{HR}=2.24)$, high-risk histology $(\mathrm{HR}=2.32)$ and tumour volume at surgery $(\mathrm{HR}=1.07 / 100 \mathrm{ml})$ remained significantly associated with an increased risk of local 
relapse. The HR for the association with biopsy had reduced in value and lost significance, from $\mathrm{HR}=1.7 ; 95 \% \mathrm{CI}: 1.21-2.37, \mathrm{p}=0.002$ in UVA to $\mathrm{HR}=1.4 ; 95 \% \mathrm{CI}: 0.9-2.17, \mathrm{p}=0.13$ in MVA (Table 4).

\section{Distant relapse risk factors}

On UVA, age $>2$ years, volume at diagnosis and at surgery, left side, high-risk tumour, metastatic status, stage III abdominal tumour, positive LN, positive margin status, biopsy and type of biopsy (PCNB and open only) were associated with an increased risk of distant recurrence (Supplemental Table 2) whereas on MVA, only age >2 years $(\mathrm{HR}=2.01)$, high-risk histology $(\mathrm{HR}=2.82)$, abdominal stage II and III ( $\mathrm{HR}=1.58$ and $\mathrm{HR}=1.52$ respectively), initial metastases $(\mathrm{HR}=2.5)$ and tumour volume at surgery $(\mathrm{HR}=1.08 / 100 \mathrm{ml})$ remained significant (Supplemental Table 2).

\section{OS and EFS of the whole cohort}

The 5-year OS/EFS for patients having no biopsy versus those having a biopsy whether we include FNA or not in the 'no biopsy' group were very similar (Supplemental Table 3).

On UVA, both OS and EFS were significantly associated with age, tumour volume at diagnosis and at surgery, metastatic status, histological risk group, pathological abdominal stage, positive LN, tumour capsule rupture, positive margin status, biopsy and type of biopsy (PCNB and open) (Supplemental Tables 4+5).

On MVA, the HR for association with biopsy lost significance for both EFS and OS (HR=1.1 and $H R=1.13$, respectively). OS remained associated with age $>4$ years $(H R=1.64)$, tumour volume at surgery $(\mathrm{HR}=1.08 / 100 \mathrm{ml})$, metastatic status $(\mathrm{HR}=3.4)$, high-risk histology $(\mathrm{HR}=4.82)$, pathological abdominal stage III $(\mathrm{HR}=2.32)$ and tumour rupture $(\mathrm{HR}=1.52)$ (Supplemental Table 4) whereas for EFS, age $>2$ years $(\mathrm{HR}=1.43)$, tumour volume at surgery 
$(\mathrm{HR}=1.08 / 100 \mathrm{ml})$, metastatic status $(\mathrm{HR}=1.91)$ and high-risk tumour $(\mathrm{HR}=2.27)$ were still significant (Supplemental Table 5).

\section{Analysis restricted to patients with no open biopsy}

The study population of this subgroup was 2910 patients because 61 patients with open biopsy were excluded. There were no differences in clinical characteristics and in chemotherapy delivered pre- and post-operatively. No other risk factors appeared in the univariate and multivariate analyses for the four different outcome variables. The HR for biopsy was similar between the whole group and the subgroup with no open biopsy (Supplemental Table 6).

\section{Analysis restricted to patients with low and intermediate risk stage I and II WT}

If we restricted the analysis to 797 patients with low and intermediate risk histology, stage I and II WT who did not receive any doxorubicin or radiotherapy, no patient had an open biopsy, 73 (9.1\%) had FNA and 180 (22.6\%) had PCNB. Patients having a biopsy (n=253) were significantly older (39.6 months versus 30.2 months in the 'no' biopsy group, $\mathrm{p}<0.001$ ) with a larger median tumour volume at diagnosis $(470 \mathrm{ml}$ versus $350 \mathrm{ml}, \mathrm{p}<0.001)$ and after pre-operative chemotherapy $(190 \mathrm{ml}$ versus $140 \mathrm{ml}, \mathrm{p}<0.018)$. Biopsy was the only significant risk factor associated with local relapse-free survival $(\mathrm{HR}=2.65$; 95\%CI: 1.28 5.45; $\mathrm{p}=0.01)$ in UVA, and remained positive in MVA (HR=2.67;95\%CI:1.07-6.67, $\mathrm{p}=0.04)$. However, if we included age as a co-factor in the MVA, since it had met the criteria to be included in all other MVA, the HR for biopsy reduced and lost statistical significance $(\mathrm{HR}=2.4 ; 95 \% \mathrm{CI}: 0.95-6.05, \mathrm{p}=0.06)($ Table 5).

\section{Discussion}


This post hoc analysis of all patients with unilateral WT treated with preoperative chemotherapy in the SIOPWT2001 trial showed that whilst there was a significant association of biopsy with local recurrence in UVA, this lost significance in MVA. The latter took account of other known factors (high-risk histology, higher tumour stage and volume, and older age at diagnosis) associated with adverse outcome that were overrepresented in the biopsied population. This finding is similar to the post-hoc analysis of the UKW3 trial but provides greater power due to the larger number of patients analyzed [6].

Although biopsy was performed routinely in the UK, in the other participating countries biopsy was recommended only when there was unusual clinical or radiological presentation. Therefore, as patients were not randomized to have a biopsy or not, the two groups were not comparable in their age, tumour size and metastatic status at diagnosis. Open biopsy appeared to be associated with a higher risk of recurrence but was only performed in a very small number of patients. The results for needle biopsy were similar for FNA and PCNB. From these results, we recommend that open biopsy should be avoided and needle biopsy reserved for cases of diagnostic uncertainty when histological confirmation is desired prior to initiating chemotherapy.

The increased risk associated with high-risk histology was substantial. This is consistent with many previous reports describing unfavorable (anaplastic) or high-risk histology (anaplastic and blastemal-type WT) as one of the strongest predictors of poor outcome in WT [7-13].

Abdominal stage III was associated with an over 1.5-fold increased risk of local recurrence but in MVA only overall survival was negatively impacted. Abdominal stage III, whatever the reason to be stage III and despite slightly different definitions of stage III between SIOP and COG, has been reported to carry a higher risk of relapse: $27 \%$ of COG and $19.6 \%$ of the 
German Society of Pediatric Oncology group stage III patients experienced a relapse [14-15]. The Italian group provided further evidence that stage III tumours due to LN metastases had a worse outcome than stage III tumours with tumour rupture and positive margins [16]. Abdominal stage III was also an independent risk factor for adverse outcome in patients with blastemal-type WT included in the SIOPWT2001 trial, along with age at diagnosis and tumour volume at surgery [17].

Larger tumour volume at diagnosis and after preoperative chemotherapy was a significant adverse factor for all outcomes but only volume after chemotherapy was significant in MVA. Tumour size was unequally distributed between both groups, a clinically expected finding also seen in the UKW3 study, where the median tumour diameter was $12.3 \mathrm{~cm}$ in the biopsy group compared to $10.7 \mathrm{~cm}$ in the no biopsy group [6].

Older age at diagnosis was an independent adverse factor for any relapse and EFS (age threshold $>2$ years) and death (age threshold $>4$ years). This is in contrast to the NWTS-4 study, where older age at diagnosis was a prognostic factor $(\mathrm{HR}=2.6)$ in UVA but failed to achieve significance when adjusted for histology and lymph node involvement [7]. Similarly in the SIOP 93-01/GPOH study, whilst median age at first diagnosis for patients experiencing a relapse was significantly older than for patients in complete continuous remission (4.5 versus 3.1 years), age lost significance in MVA [8]. However in the UKW2 and 3 trials, age older than 4 was also considered as an adverse prognostic factor in stage I favorable histology WT treated with vincristine only [18].

When we restricted the analysis to patients with stage I and II, low and intermediate risk histology tumours who receive no doxorubicin, the small number of local recurrences meant 
that the statistical power to interpret such a post hoc analysis is weak. However, in this subgroup, where chemotherapy is minimal in intensity (only vincristine and actinomycin D pre- and post-operatively) and duration (4 weeks pre-op and 4 weeks (stage I) or 27 weeks (stage II) post-operatively), it is possible that any potential association of biopsy with recurrence is stronger. Age appeared as a confounding factor for the statistical significance of biopsy in this setting, but as it was a major risk factor of survival in the other survival analyses, we included it in the MVA even though it did not reach significance in the UVA.

Limitations of the study include lack of information on potential molecular biomarkers and missing data, especially for the site of relapse, tumour rupture, tumour volume at diagnosis and pre-op. The latter was taken into account in the statistical analysis to input the missing data with either the median value for continuous covariate or with the most common value for the categorical covariate.

Hence, whilst caution is needed in interpreting our findings for clinical use, we conclude that needle biopsy (FNA or PCNB) does not clearly increase the risk of local relapse of Wilms tumour in the setting of pre-operative chemotherapy. We suggest that needle biopsy can be ignored for tumour staging purposes, providing it is done according to modern standards, under image guidance with co-axial technique. Taking account of the level of evidence of our analysis, we further recommend that needle biopsy be reserved for children whose clinical presentation or imaging are atypical for WT and where histological confirmation is desired before starting any chemotherapy [19]. The current criteria recommended by the SIOP RTSG for when biopsy should be considered are older age at diagnosis (10 years and above); presence of lung metastases in a child aged under 2 years; metastases in sites other than lung or liver; urinary infection; high serum calcium or LDH levels; and on imaging: psoas 
inflammation; presence of calcification; voluminous lymphadenopathy; no visible renal parenchyma or where the tumour appears to be almost totally extra-renal. The restriction of biopsy to these criteria will decrease the number of indications for biopsy that should be performed with FNA or PCNB. Implementation of our suggestions should be based on national and international study group consensus, treatment approach and prospective monitoring of outcomes.

\section{References}

[1] Green DM, Breslow NE, D'Angio et al. Outcome of patients with Stage II/favorable histology Wilms tumour with and without local tumour spill: a report from the National Wilms Tumour Study Group. Pediatr Blood Cancer. 2014;61:134-9.

[2] Ehrlich PF, Anderson JR, Ritchey ML, et al. Clinicopathologic findings predictive of relapse in children with stage III favorable-histology Wilms tumour. J Clin Oncol 2013;31:1196-201

[3] Pritchard-Jones K, Bergeron C, de Camargo B, et al. Omission of doxorubicin from the treatment of stage II-III, intermediate-risk Wilms' tumour (SIOP WT 2001): an openlabel, non-inferiority, randomised controlled trial. Lancet. 2015;386:1156-64

[4] Mitchell C, Pritchard-Jones K, Shannon R, et al. Immediate nephrectomy versus preoperative chemotherapy in the management of non-metastatic Wilms' tumour: results of a randomised trial (UKW3) by the UK Children's Cancer Study Group. Eur J Cancer $2006 ; 42: 2554-62$

[5] Vujanic GM, Kelsey A, Mitchell C, et al. The role of biopsy in the diagnosis of renal tumours of childhood: Results of the UKCCSG Wilms tumour study 3. Med Pediatr Oncol $2003 ; 40: 18-22$ 
[6] Irtan S, Jitlal M, Bate J, et al; Renal Tumours Committee of the Children's Cancer and Leukaemia Group (CCLG). Risk factors for local recurrence in Wilms tumour and the potential influence of biopsy - The United Kingdom experience. Eur J Cancer 2015;51:22532

[7] Shamberger RC, Guthrie KA, Ritchey ML, et al. Surgery-related factors and local recurrence of Wilms tumour in National Wilms Tumour Study 4. Ann Surg 1999;229:292-7

[8] Reinhard H, Schmidt A, Furtwangler R, et al. Outcome of relapses of nephroblastoma in patients registered in the SIOP/GPOH trials and studies. Oncol Rep 2008;20:463-7

[9] Pritchard-Jones K, Moroz V, Vujanic G, et al. Treatment and outcome of Wilms' tumour patients: an analysis of all cases registered in the UKW3 trial. Ann Oncol $2012 ; 23: 2457-63$

[10] Dome JS, Cotton CA, Perlman EJ, et al. Treatment of anaplastic histology Wilms' tumour: results from the fifth National Wilms' Tumour Study. J Clin Oncol 2006;24:2352-8

[11] Honeyman JN, Rich BS, McEvoy MP, et al. Factors associated with relapse and survival in Wilms tumour: a multivariate analysis. J Pediatr Surg 2012;47:1228-33

[12] Vujanic GM, Harms D, Sandstedt B, et al. New definitions of focal and diffuse anaplasia in Wilms tumour: the International Society of Paediatric Oncology (SIOP) experience. Med Pediatr Oncol 1999;32:317-23

[13] Graf N, van Tinteren H, Bergeron C, et al. Characteristics and outcome of stage II and III non-anaplastic Wilms' tumour treated according to the SIOP trial and study 93-01. Eur J Cancer 2012;48:3240-8

[14] Fernandez CV, Mullen EA, Chi YY, et al. Outcome and Prognostic Factors in Stage III Favorable-Histology Wilms Tumour: A Report From the Children's Oncology Group Study AREN0532. J Clin Oncol 2018;36:254-261 
[15] Graf N, Furtwängler R. Preoperative chemotherapy and local stage III in nephroblastoma. Transl Pediatr 2014;3:4-11

[16] Spreafico F, Gandola L, D'Angelo P, et al; AIEOP Wilms Tumour Working Group. Heterogeneity of disease classified as stage III in Wilms tumour: a report from the Associazione Italiana Ematologia Oncologia Pediatrica (AIEOP). Int J Radiat Oncol Biol Phys 2012;82:348-54

[17] van den Heuvel-Eibrink MM, van Tinteren H, Bergeron C, et al. Outcome of localised blastemal-type Wilms tumour patients treated according to intensified treatment in the SIOP WT 2001 protocol, a report of the SIOP Renal Tumour Study Group (SIOP-RTSG). Eur J Cancer 2015;51:498-506

[18] Pritchard-Jones K, Kelsey A, Vujanic G, et al. Older age is an adverse prognostic factor in stage I, favorable histology Wilms' tumour treated with vincristine monochemotherapy: a study by the United Kingdom Children's Cancer Study Group, Wilm's Tumour Working Group. J Clin Oncol 2003;21:3269-75

[19] Jackson TJ, Williams RD, Brok, J, et al, on behalf of the Children's Cancer and Leukaemia Group (CCLG) Renal Tumours Group. The diagnostic accuracy and clinical utility of pediatric renal tumor biopsy: Report of the UK experience in the SIOP UK WT2001 trial. Ped Blood Cancer 2019 in press. DOI: 10.1002/pbc.27627 


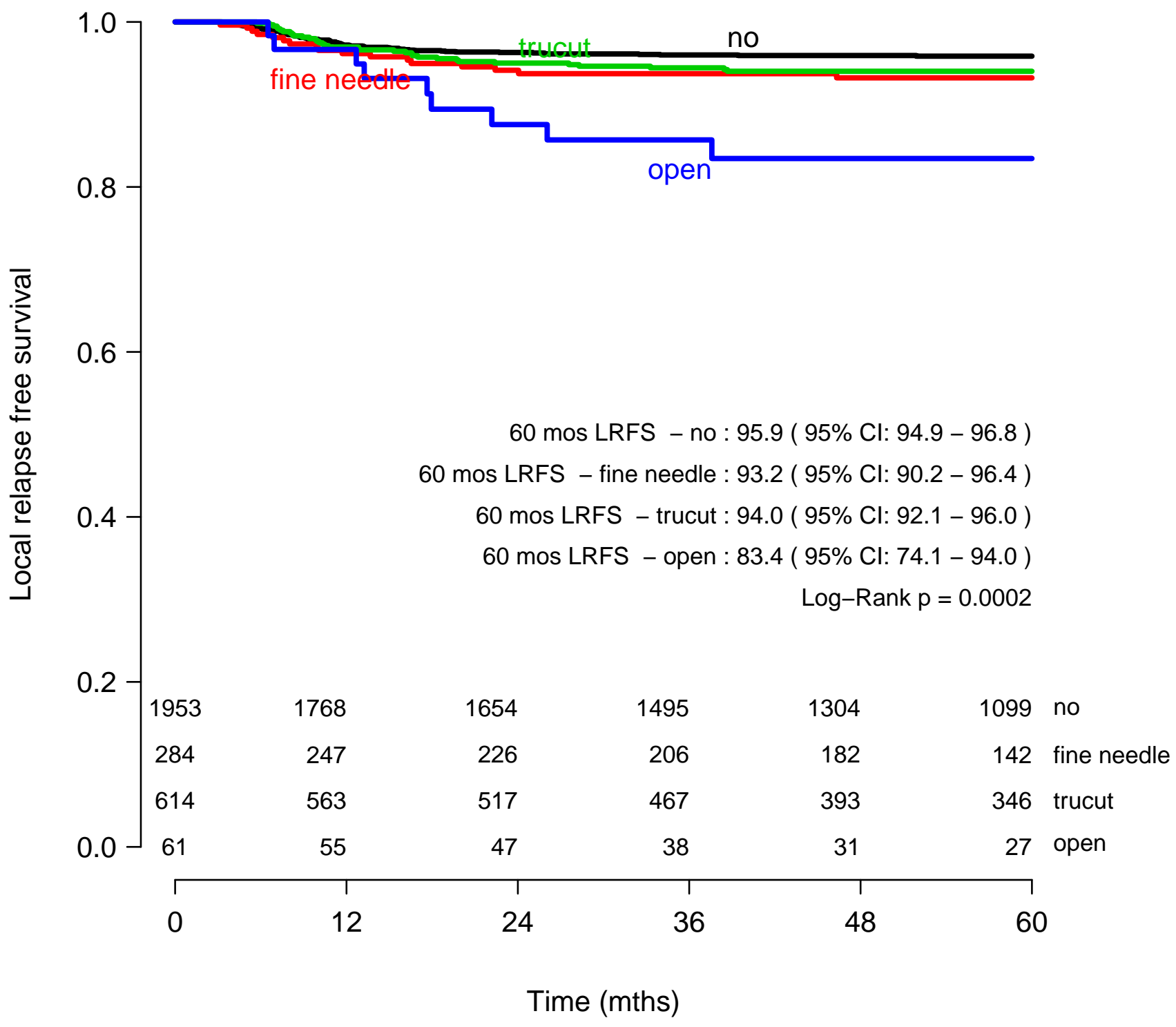




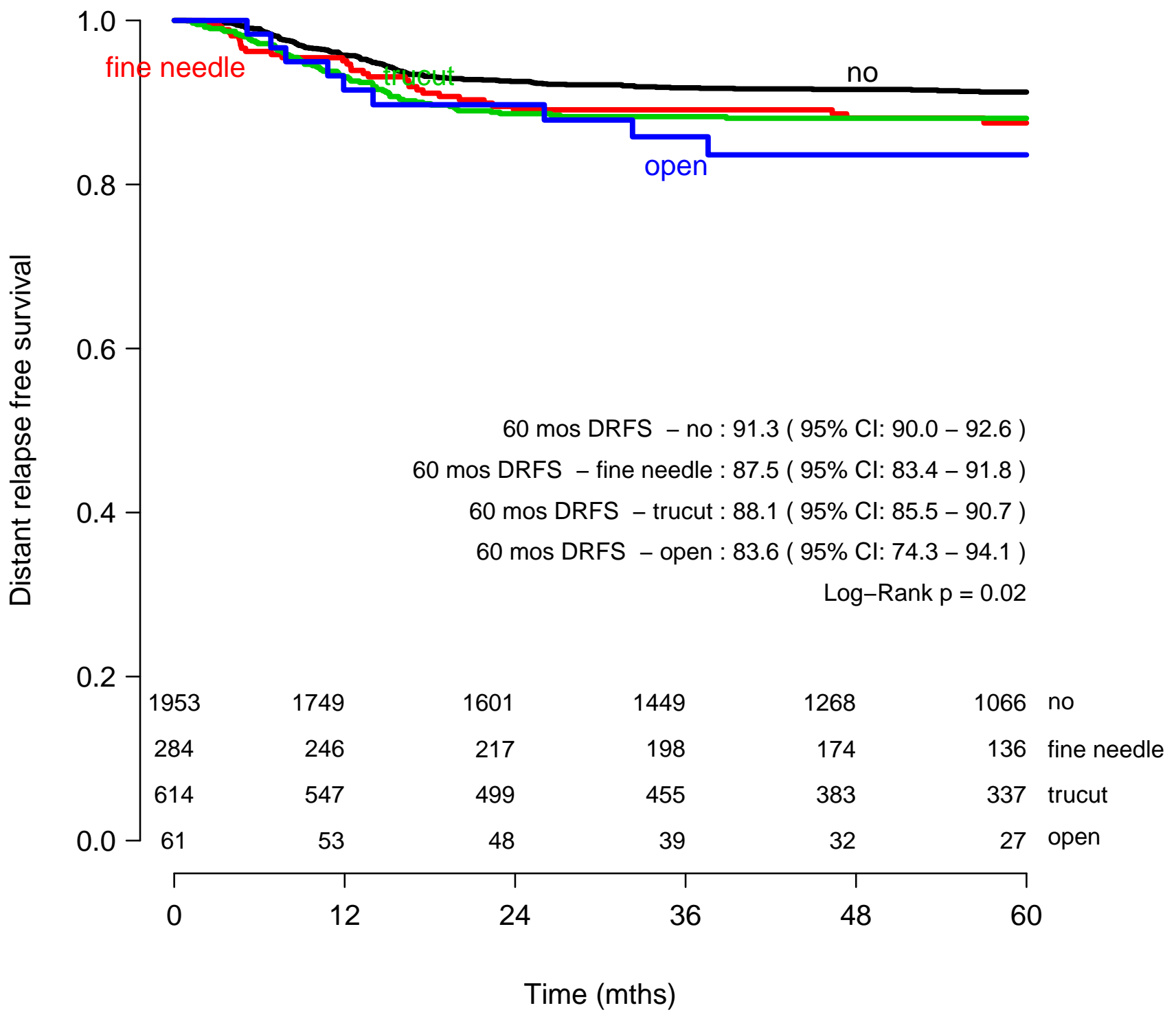


Table 1

Biopsy type by national centre

\begin{tabular}{|c|c|c|c|c|c|c|c|c|}
\hline \multirow[t]{2}{*}{ Group } & \multicolumn{2}{|c|}{ No biopsy } & \multicolumn{2}{|c|}{ FNA } & \multicolumn{2}{|c|}{ PCNB } & \multicolumn{2}{|c|}{ Open biopsy } \\
\hline & No. & $\%$ & No. & $\%$ & No. & $\%$ & No. & $\%$ \\
\hline Total & 2,002 & 67 & 288 & 10 & 620 & 21 & 61 & 2 \\
\hline Brazil & 257 & 79 & 29 & 9 & 30 & 8 & 8 & 3 \\
\hline Germany & 694 & 92 & 60 & 8 & 0 & 0 & 0 & 0 \\
\hline France & 477 & 79 & 78 & 13 & 39 & 6 & 12 & 2 \\
\hline SIOP-NL & 558 & 74 & 87 & 12 & 92 & 12 & 14 & 2 \\
\hline UK & 16 & 3 & 34 & 6 & 459 & $* 86$ & 27 & 5 \\
\hline
\end{tabular}

* UK continued its established national practice from the UKW3 trial of recommending percutaneous cutting needle biopsy (PCNB) prior to chemotherapy in all patients. FNA: fine needle aspirate. 
Table 2

Patient and tumour characteristics by biopsy group.

\begin{tabular}{|c|c|c|c|}
\hline Biopsy & No & Yes & $\mathrm{P}$ values \\
\hline Total & 2002 & 969 & \\
\hline Sex & & & 0.7 \\
\hline Male & $933(46.7 \%)$ & $444(45.9 \%)$ & \\
\hline Female & $1065(53.3 \%)$ & $524(54.1 \%)$ & \\
\hline Age & & & $<0.001$ \\
\hline $0.5-1.99$ & $567(28.3 \%)$ & $192(19.8 \%)$ & \\
\hline $2-3.99$ & $718(35.9 \%)$ & $317(32.7 \%)$ & \\
\hline $4-17.5$ & $717(35.8 \%)$ & $460(47.5 \%)$ & \\
\hline Category & & & $<0.001$ \\
\hline Localized & $1752(87.5 \%)$ & $777(80.2 \%)$ & \\
\hline Metastatic & $250(12.5 \%)$ & $192(19.8 \%)$ & \\
\hline Side & & & 0.187 \\
\hline Right & $953(47.6 \%)$ & $487(50.3 \%)$ & \\
\hline Left & $1049(52.4 \%)$ & $482(49.7 \%)$ & \\
\hline $\begin{array}{l}\text { Volume at diagnosis }(\mathbf{m l}) \\
\text { (Median \& IQR) }\end{array}$ & $410(220-670)$ & $570(310-880)$ & $<0.001$ \\
\hline $\begin{array}{l}\text { Volume at surgery (ml) } \\
\text { (Median \& IQR) }\end{array}$ & $140(50-300)$ & $180(70-410)$ & $<0.001$ \\
\hline Abdominal stage & & & $<0.001$ \\
\hline $\begin{array}{l}\text { I } \\
\text { II }\end{array}$ & $\begin{array}{l}1025(53.6 \%) \\
455(23.8 \%)\end{array}$ & $\begin{array}{l}374(40.0 \%) \\
227(24.3 \%)\end{array}$ & \\
\hline III & $433(22.6 \%)$ & $334(35.7 \%)$ & \\
\hline Margin status & & & $<0.001$ \\
\hline Negative & $1782(89.0 \%)$ & $766(79.1 \%)$ & \\
\hline Positive & $220(11.0 \%)$ & $203(20.9 \%)$ & \\
\hline Lymph node status & & & $<0.001$ \\
\hline Negative & $1793(89.6 \%)$ & $817(84.3 \%)$ & \\
\hline Positive & $209(10.4 \%)$ & $152(15.7 \%)$ & \\
\hline Tumour rupture & & & 0.004 \\
\hline $\begin{array}{l}\text { No } \\
\text { Yes }\end{array}$ & $\begin{array}{l}1339(91.1 \%) \\
131(8.9 \%)\end{array}$ & $\begin{array}{l}739(87.2 \%) \\
108(12.8 \%)\end{array}$ & \\
\hline $\begin{array}{l}\text { Histological Risk group } \\
\text { (panel review/local pathol.) }\end{array}$ & & & 0.027 \\
\hline Low Risk & $111(5.8 \%)$ & $54(5.9 \%)$ & \\
\hline Intermediate Risk & $1558(81.9 \%)$ & $731(78.3 \%)$ & \\
\hline High Risk & $234(12.3 \%)$ & $149(15.8 \%)$ & \\
\hline
\end{tabular}


Table 3

Relapses and deaths by biopsy group

\begin{tabular}{llll}
\hline & $\begin{array}{l}\text { No Biopsy } \\
(\mathbf{n = 2 0 0 2})\end{array}$ & $\begin{array}{l}\text { Biopsy } \\
(\mathbf{n = 9 6 9 )}\end{array}$ & $\begin{array}{l}\text { Total } \\
(\mathbf{n = 2 9 7 1})\end{array}$ \\
\hline Local relapse only & $45(2.2 \%)$ & $41(4.2 \%)$ & $86(2.9 \%)$ \\
Combined relapse & $31(1.5 \%)$ & $22(2.3 \%)$ & $53(1.8 \%)$ \\
\hline All Local relapses & $\mathbf{7 6}(\mathbf{3 . 8 \%})$ & $\mathbf{6 3}(\mathbf{6 . 5 \%})$ & $\mathbf{1 3 9}(\mathbf{4 . 7 \%})$ \\
Distant relapse only & $131(6.5 \%)$ & $91(9.4 \%)$ & $222(7.5 \%)$ \\
\hline Unknown site & $49(2.4 \%)$ & $10(1.0 \%)$ & $59(2.0 \%)$ \\
\hline Any relapse & $\mathbf{2 5 6 ( 1 2 . 8 \% )}$ & $\mathbf{1 6 4}(\mathbf{1 6 . 9 \%})$ & $\mathbf{4 2 0}(\mathbf{1 4 . 1 \%})$ \\
\hline Death & $107(5.3 \%)$ & $97(10.0 \%)$ & $204(6.9 \%)$ \\
\hline
\end{tabular}


Table 4

Univariate and multivariable analysis of risk factors of recurrence for any local relapse.

\begin{tabular}{|c|c|c|c|c|c|c|c|c|}
\hline & & \multirow{2}{*}{$\begin{array}{l}\text { No. with } \\
\text { local } \\
\text { relapse }\end{array}$} & \multicolumn{3}{|c|}{$\begin{array}{c}\text { Univariate } \\
\text { analysis }\end{array}$} & \multicolumn{3}{|c|}{$\begin{array}{c}\text { Multivariable } \\
\text { analysis }\end{array}$} \\
\hline & & & HR & $95 \% \mathrm{CI}$ & $p$-value & $\mathrm{HR}$ & $95 \% \mathrm{CI}$ & $p$-value \\
\hline \multirow{2}{*}{ Biopsy } & No & 76 & 1 & & 0.002 & 1 & & 0.13 \\
\hline & Yes & 63 & 1.70 & $1.21-2.37$ & & 1.40 & $0.9-2.17$ & \\
\hline Age & $0.5-1.99$ & 14 & 1 & & $<0.001$ & 1 & & \\
\hline \multirow[t]{2}{*}{ (years) } & $2-3.99$ & 46 & 2.37 & $1.30-4.32$ & & 2.24 & $1.22-4.09$ & 0.01 \\
\hline & $4-17.5$ & 79 & 3.60 & $2.04-6.36$ & & 2.78 & $1.55-4.99$ & 0.001 \\
\hline \multirow[t]{2}{*}{ Risk } & $\begin{array}{c}\text { Low + } \\
\text { Intermediate }\end{array}$ & 100 & 1 & & $<0.0001$ & 1 & & \\
\hline & High & 39 & 2.83 & $1.95-4.1$ & & 2.32 & $1.58-3.42$ & $<0.0001$ \\
\hline $\begin{array}{c}\text { Volume at } \\
\text { surgery }\end{array}$ & $(/ 100 \mathrm{ml})$ & 139 & 1.11 & $1.07-1.16$ & $<0.0001$ & 1.07 & $1.02-1.12$ & 0.01 \\
\hline $\begin{array}{l}\text { Volume at } \\
\text { diagnosis }\end{array}$ & $(/ 100 \mathrm{ml})$ & 139 & 1.09 & $1.06-1.13$ & $<0.0001$ & & & \\
\hline Lymph node & Negative & 114 & 1 & & 0.03 & & & \\
\hline status & Positive & 25 & 1.60 & $1.03-2.46$ & & & & \\
\hline Tumour & No & 119 & 1 & & 0.004 & & & \\
\hline Rupture* & Yes & 20 & 1.96 & $1.22-3.15$ & & & & \\
\hline Abdominal & I & 59 & 1 & & 0.06 & & & \\
\hline \multirow[t]{2}{*}{ stage } & II & 32 & 1.14 & $0.74-1.76$ & & & & \\
\hline & III & 48 & 1.57 & $1.07-2.30$ & & & & \\
\hline \multirow[t]{2}{*}{ Site } & Right & 67 & 1 & & 0.94 & & & \\
\hline & Left & 72 & 1.01 & $0.72-1.41$ & & & & \\
\hline Margin & Negative & 117 & 1 & & 0.62 & & & \\
\hline status & Positive & 22 & 1.12 & $0.71-1.76$ & & & & \\
\hline \multirow[t]{2}{*}{ Gender } & Male & 61 & 1 & & 0.57 & & & \\
\hline & Female & 78 & 1.10 & $0.78-1.54$ & & & & \\
\hline \multirow[t]{2}{*}{ Category } & Localized & 114 & 1 & & 0.25 & & & \\
\hline & Metastatic & 25 & 1.28 & $0.83-1.98$ & & & & \\
\hline \multirow[t]{4}{*}{ Biopsy done } & no & 76 & 1 & & 0.0002 & & & \\
\hline & Fine needle & 17 & 1.61 & $0.95-2.74$ & & & & \\
\hline & Trucut & 37 & 1.52 & $1.03-2.26$ & & & & \\
\hline & Open & 9 & 3.92 & $1.96-7.83$ & & & & \\
\hline
\end{tabular}

* in a sensitivity analysis in which the value of 'yes' rather than 'no' was imputed for the patients with missing data for 'rupture at surgery', the hazard ratio and $\mathrm{p}$ value for any 
association of local recurrence with biopsy were 1.175 and 0.37 , respectively, adding further weight to the lack of significant association of this parameter in multi-variable analysis. 


\section{Table 5}

Univariate and multivariable analysis of risk factors for local recurrence restricted to patients with intermediate risk stage I and II WT that received no doxorubicin and no radiotherapy $(\mathrm{n}=797)^{*}$

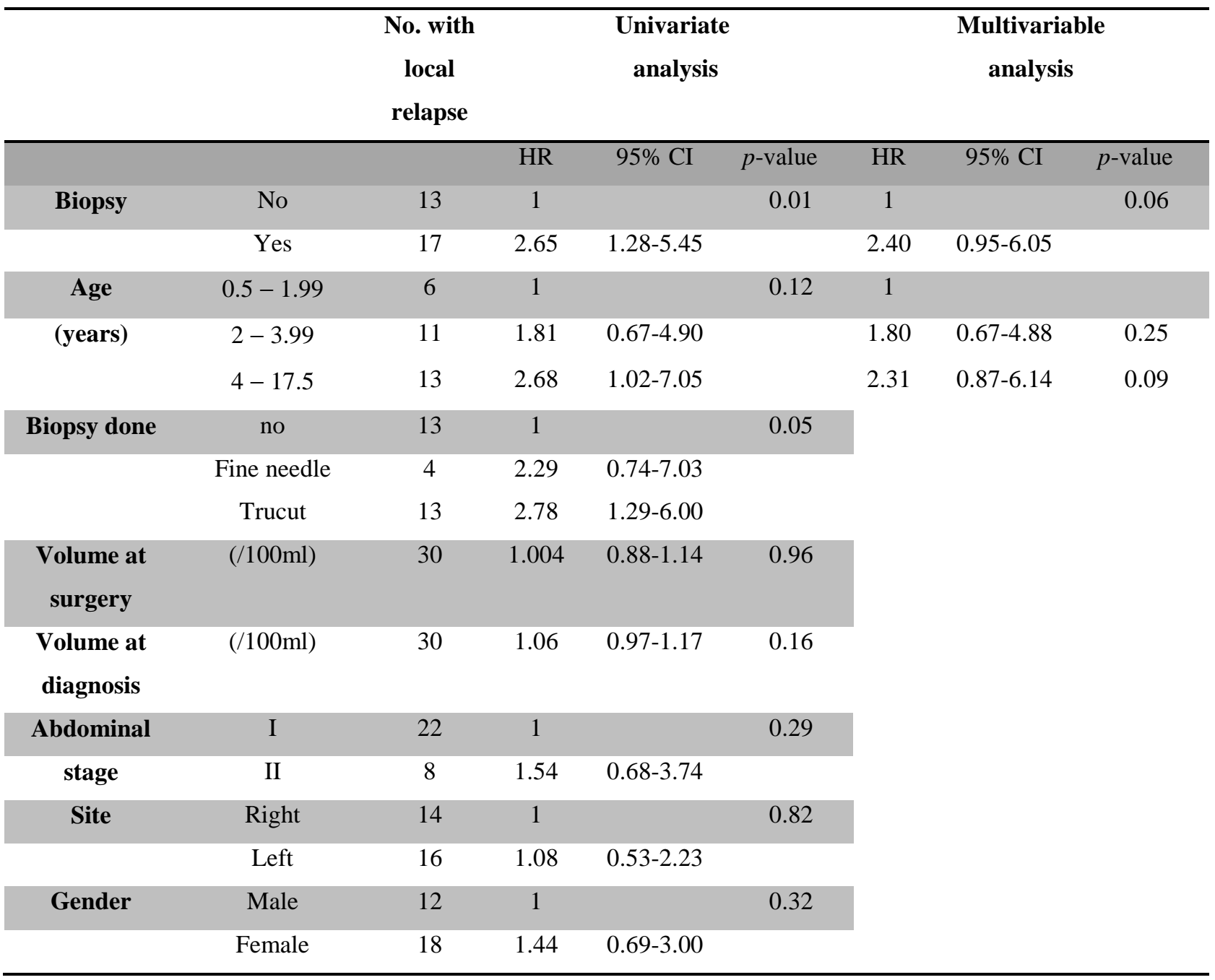

* This cohort excluded any patients documented to have had open biopsy, positive lymph nodes, tumour capsule rupture and those who received radiotherapy to the flank or 'high-risk' post-operative chemotherapy regimens. 